\title{
Performance Analysis of Grey Level Fitting Mechanism based Gompertz Function for Image Reconstruction Algorithms in Electrical Capacitance Tomography Measurement System
}

\author{
Josiah Nombo \\ Department of Electronics and \\ Telecommunication \\ Engineering, Nelson Mandela \\ African Institution of Science \\ and Technology, Arusha, \\ Tanzania
}

\author{
Alfred Mwambela \\ Department of Electronics and \\ Telecommunication \\ Engineering, University of Dar \\ es Salaam, Dar es Salaam, \\ Tanzania
}

\author{
Michael Kisangiri \\ Department of Electronics and \\ Telecommunication \\ Engineering, Nelson Mandela \\ African Institution of Science \\ and Technology, Arusha, \\ Tanzania
}

\begin{abstract}
This paper analyses the performance of grey level fitting mechanism based on Gompertz function used in Electrical Capacitance Tomography measurement system. In order to evaluate its performance, the data fitting mechanism has been applied to common image reconstruction algorithms which include; Linear Back Projection, Singular Value Decomposition, Tikhonov Regularization, Iterative Tikhonov Regularization, Landweber iteration and Projected Landweber iteration. Images were reconstructed using measured capacitance data for annular and stratified flows, and qualitative and quantitative evaluation were done on the reconstructed images in comparison with respective reference images. Results show that this grey level fitting mechanism is better in terms of improving image spatial resolution, minimizing relative image error and distribution error and maximizing correlation coefficient.
\end{abstract}

\section{General Terms}

Process Tomography, Image Reconstruction Algorithms.

\section{Keywords}

Electrical Capacitance Tomography, Image Reconstruction Algorithms, Data Fitting, Gompertz function.

\section{INTRODUCTION}

Electrical capacitance tomography (ECT) is an industrial flow imaging technique which has been fast developed in recent years[1-3]. Because of its distinct advantages such as simple structure, wide application field, low cost, non-destructive non-invasive and better safety, it has been the most popular and attractive research direction in flow imaging technique[4]. However, it is very difficult to reconstruct high quality images using ECT systems. This difficulty is caused by non-linearity between measured capacitance and permittivity distribution, soft-field effect of ECT sensor, limited number of measurements, and severe ill-condition caused by measurement errors [5,6]. Thus, image reconstruction has always been the main challenge in practical application and development of ECT measurement method[7,8].

Therefore, exploring good image reconstruction algorithms is important. Presently, image reconstruction methods used can be categorized into two groups namely; non iterative and iterative image reconstruction methods. The most common non iterative methods include Linear Back Projection (LBP), Singular Value Decomposition (SVD)[9] and Tikhonov regularization methods. Iterative image reconstruction algorithms include Landweber iterative method and its variations[10-12], Conjugate Gradient method[5] and artificial neural networks and genetic algorithms[13-15], but the most commonly used is Landweber iteration.

The LBP algorithm has been extensively applied in ECT image reconstruction because of its high reconstruction speed but it introduces large error in image reconstruction. The standard Tikhonov regularization method is one of the best method to solve the ill-posed problem, but in ECT it tends to generate a smooth approximation solution, in that case it can lead the lost detailed information which in turn result low image spatial resolution.

Landweber algorithm has good image reconstruction quality. However, it has the disadvantage of slow convergence therefore it not suitable for real-time imaging environment. Artificial neural network and genetic algorithms are intelligent algorithms which simulate biological evolution and genetic. These algorithms select set of data with the best fitness through the selection and evolution of the initial estimate. Then use selected data as the optimal solution of the algorithm. However, these algorithms has the problem of early convergence. This result in premature loss of the population diversity, and causes the algorithm to fall into locally optimal solution and hence cannot find the global optimal solution.

This paper investigates the performance of Gompertz based data fitting algorithm using common selected image reconstruction algorithms for image reconstruction in ECT. Experimental results shows that data fitting using Gompertz function proved to produce better images in terms image spatial resolution.

\section{LITERATURE REVIEW}

\subsection{Basic Structure of ECT System}

Typical ECT system consist of main three components (Fig. 1); a multi-electrode sensor, the sensing electronics circuit for data acquisition and a computer system for image reconstruction, data storage, interpretation and display[1]. 


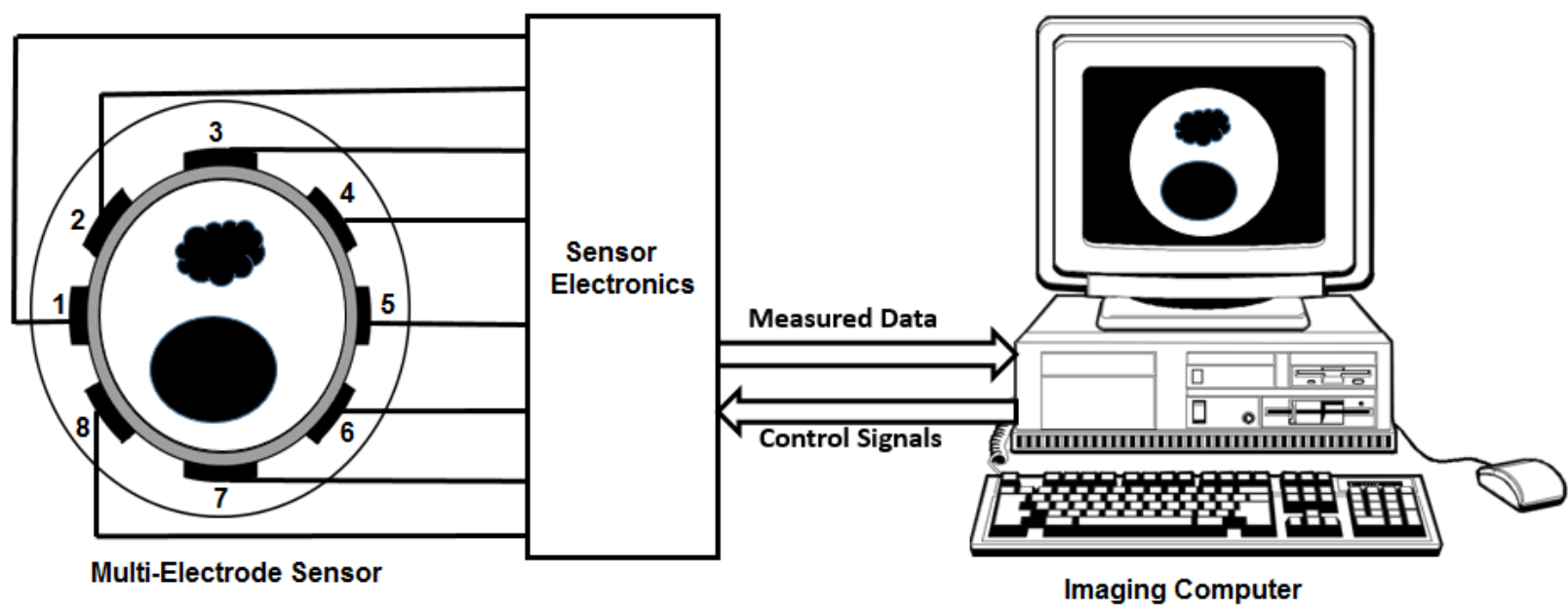

Fig. 1. Typical ECT System with an Eight Electrode Sensor

A set of electrode are mounted around a pipe or vessel and capacitance between all single electrode combinations are measured. The sensing electronics achieve the data acquisition. The computer controls the system hardware and implements image reconstruction that shows the permittivity distribution.

In ECT image reconstruction there are two main computational aspects: the forward problem and the inverse problem. The forward problem involves calculation of capacitances from given permittivity distribution and boundary conditions. The inverse problem involves estimation of permittivity distribution from measured capacitance data. Obtained permittivity distributions are usually presented as a visual image and hence the process is called image reconstruction. Simplified image reconstruction model is usually represented as[1]:

$$
\mathrm{SG}=\mathrm{C}
$$

where $C$ is an $m \times 1$ dimensional vector representing normalized capacitances values, $G$ is an $n \times 1$ dimensional vector representing normalized permittivity distribution also it represents grey levels values, $S$ an $m \times n$ matrix representing sensitivity distribution.

There are three main difficulties with image reconstruction in ECT[1]. First, the non- linear relationship between permittivity distribution and measured capacitance and the electric field is distorted by the material being monitored, this is called "soft field" effect. Second, the number of independent measurement capacitance data is normally fewer than the number of pixels required to reconstruct an image. And third the inverse problem is ill-posed and ill-conditioned. Therefore the ECT solution is often unstable and sensitive to noise in the input data.

The task of inverse problem (image reconstruction) is to find $\mathrm{G}$ rapidly and efficiently from the known $S$ and $C$ parameters.

\subsection{Common reconstruction Algorithm}

Different approaches have been proposed to reconstruct images using ECT System, but the most commonly used include: LBP, SVD, Tikhonov, Iterative Tikhonov, and Landweber iteration. In this section a brief description of these methods is given.

\subsubsection{Linear Back Projection}

LBP was the first and still the most common algorithm used to reconstruct image from ECT systems[16,17]. In LBP algorithm it is assumed that sensitivity is constant within and between sensitivity areas. The relationship between permittivity distribution and the measured capacitance for a given sensitivity matrix is given by

$$
G(k)=\frac{\sum_{i=1}^{n-1} \sum_{j=i+1}^{n} S_{i j}(k) C_{i j}}{\sum_{i=1}^{n-1} \sum_{j=i+1}^{n} S_{i j}(k)}
$$

where $n$ is the number of electrodes, $S_{i j}(k)$ is the sensitivity at position $k$ when measuring capacitance between $i j$ electrode pair.

The drawback of LBP algorithm is that the algorithm smears out sharp transition in the dielectric constant distribution. To improve the contrast between high and low permittivity distribution materials it is necessary to further process the obtained permittivity distribution to estimate new grey levels.

\subsubsection{Singular Value Decomposition}

SVD is a technique used in solving systems of equations where by matrix is factorized into three new matrices which are then used to find a solution to the system of equations. In ECT the technique has been used to calculate grey levels [18]. In this approach the sensitivity matrix $\mathrm{S}$ is decomposed into three matrices and then used to solve (1) as follows:

$$
S=\mathrm{U} \Sigma \mathrm{V}^{T}
$$

where $U$ is a $m \times m$ real or complex unitary matrix, $\sum$ is a $m \times n$ rectangular diagonal matrix with non-negative real numbers on the diagonal, and $\mathrm{V}^{T}$ (the conjugate transpose of $\mathrm{V}$ ) is an $n \times n$ real or complex unitary matrix. The diagonal entries $\sum_{i i}$ of $\sum$ are known as the singular values of $S$. The $m$ columns of Uand the $n$ columns of Vare called the leftsingular vectors and right-singular vectors of $S$, respectively. Using SVD the solution to (1) can now be expressed as

$$
G=\mathrm{v} \sum^{T} \mathrm{U} C
$$

where $G$ is a $n \times m$ diagonal matrix, however, the solution is not unique because the inverse problem is ill posed and underdetermined.

\subsubsection{Tikhonov, and Iterative Tikhonov}

Standard Tikhonov regularization is an effective method to deal with ill-posed problems. The essence of the method is to 
transform the solving of equation (1) into an optimization problem [19].

$$
\operatorname{minJ}(\mathrm{G})=\|\mathrm{SG}-\mathrm{C}\|^{2}+\alpha\|G\|^{2}
$$

where $\alpha>0$, is called the regularization parameter, $\|G\|^{2}$ is a stabilizing item . Minimizing (5) gives a solution to (1) as

$$
\mathrm{G}=\left(S^{T} S+\alpha I\right)^{-1} S^{T} C
$$

When standard Tikhonov is used to in image reconstruction in ECT it reconstruct images with quality far from perfect. The main disadvantage of this method is its excessive smoothness[20].This effect removes detailed information from reconstructed image and hence decrease spatial resolution.

The drawbacks of standard Tikhonov algorithms can be improved by finding the solution in iterative manner using

$\widehat{G}_{k+1}=\widehat{G}_{k}-\left(S^{T} S+\mu I\right)^{-1} S^{T}\left(S \widehat{G}_{k}-C\right)$

Equation (7) is called iterative Tikhonov regularization. Iterative Tikhonov method, and is the one which has been used in image reconstruction in ECT.

The main drawback of iterative Tikhonov algorithm is its intensive computational cost compared to other iterative algorithms.

\subsubsection{Landweber and Projected Landweber iteration}

Landweber iteration algorithm has been widely adopted in recent years. In principle, the algorithm uses steepest descent method to minimize the following objective function

$$
\operatorname{minf}(\mathrm{G})=\frac{1}{2}|| \mathrm{SG}-\mathrm{C} \|^{2}
$$

Minimizing (8) gives a solution to (1) as

$$
\widehat{G}_{k+1}=\widehat{G}_{k}-\alpha S^{T}\left(S \widehat{G}_{k}-C\right)
$$

From the point of view of numerical optimization, Landweber iteration algorithm is only steepest descent algorithm, and the rate of convergence is relatively slow. Another drawback of Landweber iteration is that it converges to the local minimum, this behavior is called semi-convergence. This means that the image error decrease fast at the beginning of the iteration but it increases after reaching the minimum point as the iteration continues. Different methods have been proposed to improve the Landweber iteration algorithm[21].

One of the approach that has been used to improve Landweber algorithms is by using, a projection operator $P$. This operator improve the overall convergence property of Landweber iteration. The projected algorithm is called Projected Landweber iteration represented by

$$
\left.\widehat{G}_{k+1}=\widehat{P\left[G_{k}\right.}-\alpha S^{T}\left(S \widehat{G}_{k}-C\right)\right]
$$

Where projection operator $\mathrm{P}$ is defined by

$$
P[f(x)]=\left\{\begin{array}{llr}
0 & \text { if } & f(x)<0 \\
f(x) & \text { if } & 0 \leq f(x) \leq 1 \\
1 & \text { if } & f(x)>1
\end{array}\right.
$$

The projection operator make sure that reconstructed images are non-negative and upper bounded. In general projected Landweber algorithm is faster compared to other iteration algorithms, because it uses first order derivative. However, it needs many iterations to reach a minimum point.

\subsubsection{Grey Level Fitting based on Gompertz}

Fitting Function

In ECT grey level fitting using Gompertz function was proposed by[22]. The proposed model for image reconstruction unit using gompertz function grey level fitting consist of three main parts which include reconstruction stage, data fitting stage and display stage is as shown in Fig. 2

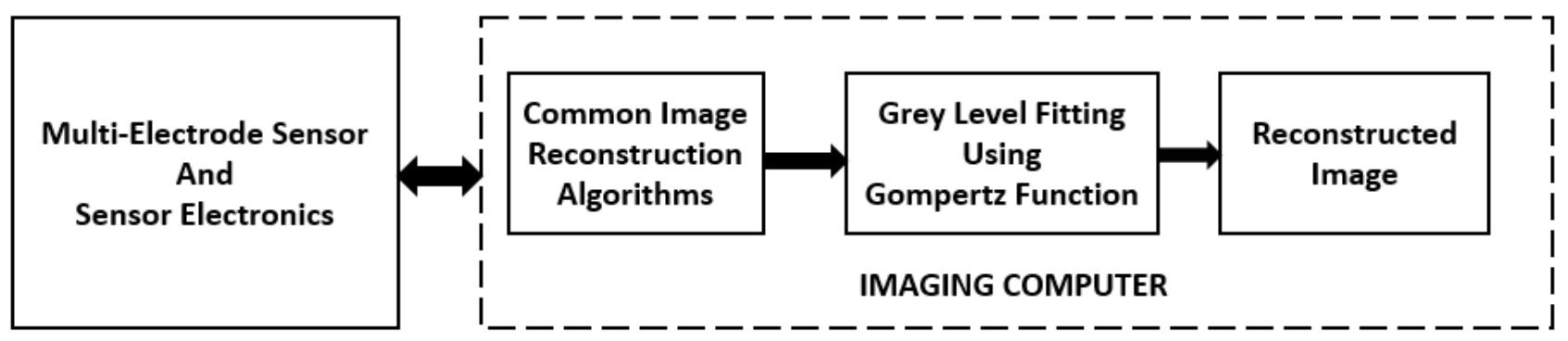

Fig. 2. ECT Image Reconstruction Stage incorporating Gompertz Data Fitting Mechanism

Reconstruction stage is implemented using common image reconstruction algorithms such as LBP, SVD, Tikhonov regularization, Landweber iteration or Projected Landweber iteration. Data fitting stage is implemented using gompertz function as:

$$
\widehat{\mathrm{G}}_{\mathrm{k}}\left(\mathrm{G}_{\mathrm{k}} ; \theta_{1}, \theta_{2}\right)=\mathrm{e}^{-\theta_{1} \mathrm{e}^{-\theta_{2} \mathrm{G}(\mathrm{k})}}
$$

where $\hat{G}_{k}$ represent fitted grey levels, $G_{k}$ represent reconstructed grey levels, $\left(\theta_{1}, \theta_{2}\right)$ are fitting parameters.

Parameters $\theta_{1}$ and $\theta_{2}$ in (12) are calculated Least Square method(LSM) $[21,23,24]$. The detailed procedure for calculating $\left(\theta_{1}, \theta_{2}\right)$ can be found in[22]. In the display stage the fitted grey levels are drawn and displayed as images.

\section{METHODOLOGY}

Experiments were carried out on annular and stratified flows using oil gas distribution, using an 8-electrode capacitance sensor and images were reconstructed using reconstruction algorithms implemented using Matlab ${ }^{\mathrm{TM}}$. Six common image reconstruction algorithms namely: LBP, SVD, Tikhonov Regularization, Iterative Tikhonov Regularization, Landweber iteration and Projected Landweber iteration were used were used to reconstruct initial images from measured capacitance data. The initial images were then used to find estimate images using Gompertz data fitting mechanism, and the results were compared with initial images and reference images. The following criteria were used in the evaluation process. 
i. Relative Image Error (RIE): Because images are treated as vectors, their norms were used to calculate the relative error [1]. The smaller the percentage RIE the better the quality

$$
\mathrm{RIE}=\frac{\|\widehat{\mathrm{G}}-\mathrm{G}\|}{\|\mathrm{G}\|}
$$

ii. Distribution Error (DE): The average of total sum of the absolute difference in grey level values between the test (reference) image and the filtered image [25].

$$
D E=\frac{1}{n} \sum_{i=1}^{n}\left|\widehat{G_{I}}-G_{I}\right|
$$

iii. Correlation Coefficient (CC) between the reference image and the reconstructed image [26]

$$
\mathrm{CC}=\frac{\sum_{\mathrm{i}=1}^{\mathrm{n}}\left(\widehat{\mathrm{G}_{\mathrm{i}}}-\widehat{\mathrm{G}}\right)\left(\mathrm{G}_{\mathrm{i}}-\overline{\mathrm{G}}\right)}{\sqrt{\sum_{\mathrm{i}=1}^{\mathrm{n}}\left(\widehat{\mathrm{G}_{\mathrm{i}}}-\overline{\mathrm{G}}\right)^{2} \sum_{\mathrm{i}=1}^{\mathrm{n}}\left(\mathrm{G}_{\mathrm{i}}-\overline{\mathrm{G}}\right)^{2}}}
$$

Images with minimum image error, minimum distribution error, and high correlation coefficient indicate best results.

\section{EXPERIMENTAL RESULTS AND DISCUSSION}

This section presents a summary of experimental results for qualitative evaluation together with the comparison made between different reconstruction algorithms. Quantitative comparison results using RIE, DE, and $\mathrm{CC}$ are also given. These criteria were used to determine whether a given algorithm provide an image close to true image. Fig. 2 to Fig. 7 presents qualitative results of the six selected common algorithms and their modification using gompertz function.

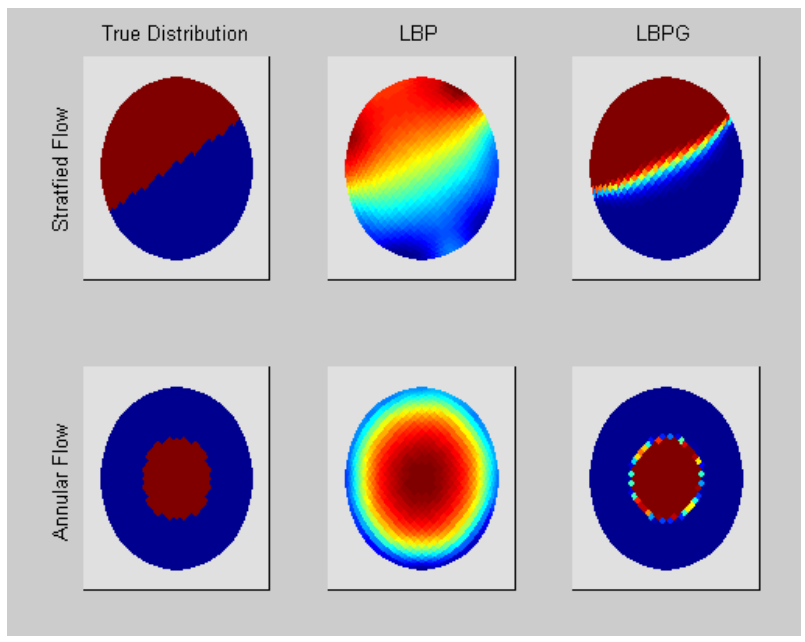

Fig 3: Image Reconstructed Using LBP Algorithm

Fig. 3 shows image reconstructed using the LBP algorithm and its modified version LBPG. From the reconstructed images it can be seen that there is significant improvement in image quality for the image reconstructed using LPBG compared to LBP.

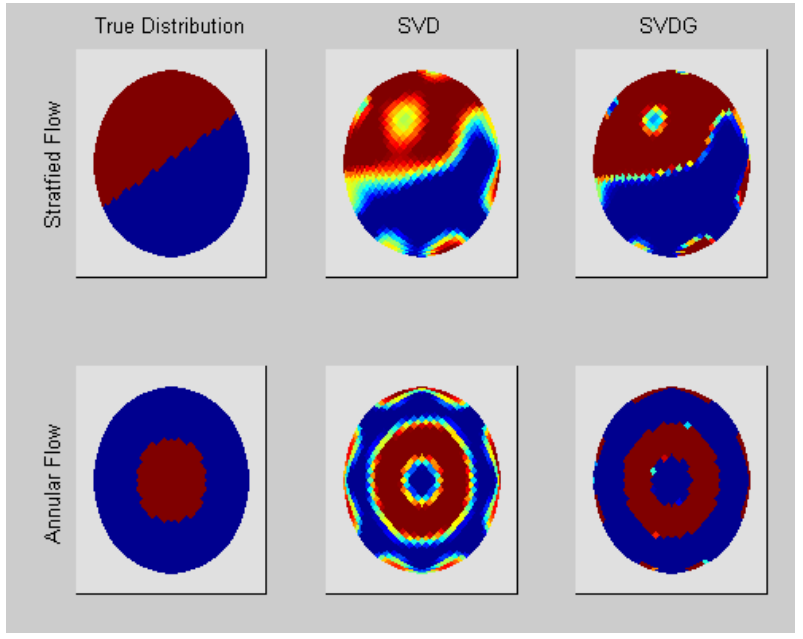

Fig 4: Image Reconstructed Using SVD Algorithm

Fig.4 presents reconstructed images using SVD and its modified version (SVDG). Results indicates image produced by SVDG are highly distorted at the center for both cases of annular and stratified flows. But the effect is more vivid for the case of annular flow. This suggest that the combination of singular value decomposition and Gompertz based fitting does not produce best results

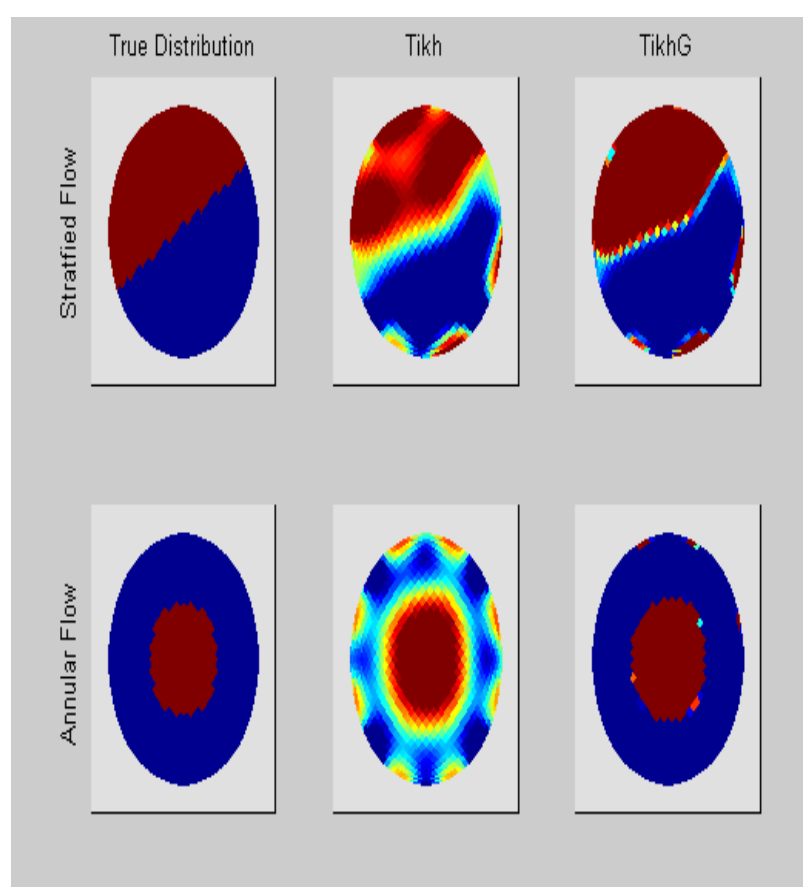

Fig 5: Image Reconstructed Using Tikhonov Algorithm

Fig. 5 shows results from Tikhonov (Tikh) regularization algorithms and its modification using Gompertz based fitting (TikhG). TikhG provide superior images compared to Tikh for both case of annular and stratified flows. 


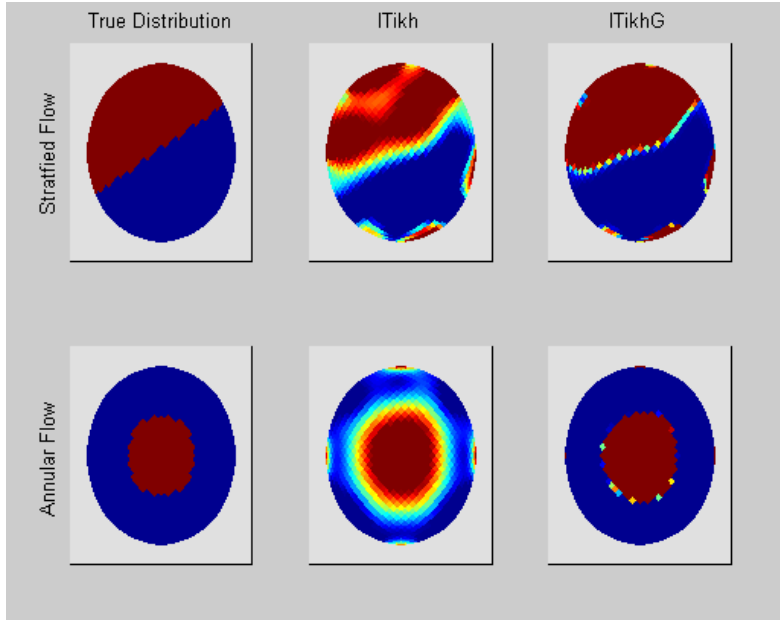

Fig 6: Image Reconstructed Using Iterative Tikhonov Algorithm

Reconstructed images using iterative Tikhonov (ITikh) and its modification (ITikhG) are given in Fig. 6. Again as in Tikhonov algorithms ITikhG produce images with remarkable quality compared to ITikh for both annular and stratified flows.

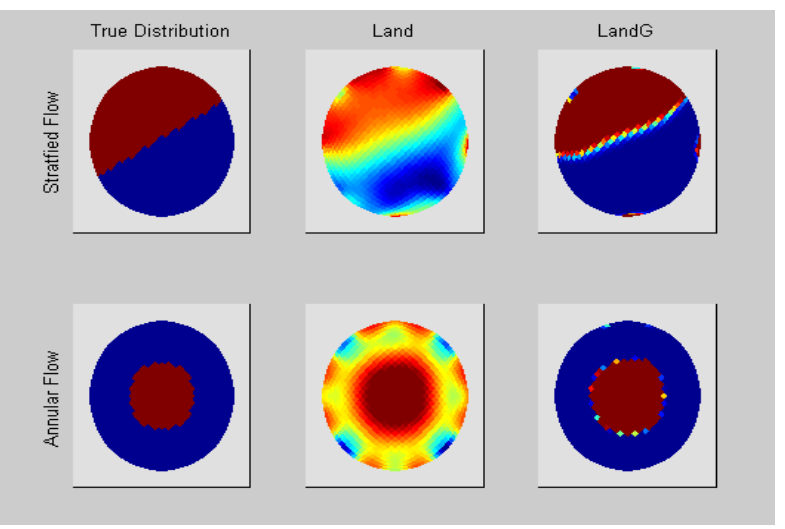

Fig 7: Image Reconstructed Using Landweber Algorithm
Fig. 7 shows results for image reconstructed using the normal Landweber iteration algorithm (Land) and its modification (LandG). It can be seen clearly that, LandG produce good image compared to Land algorithm.

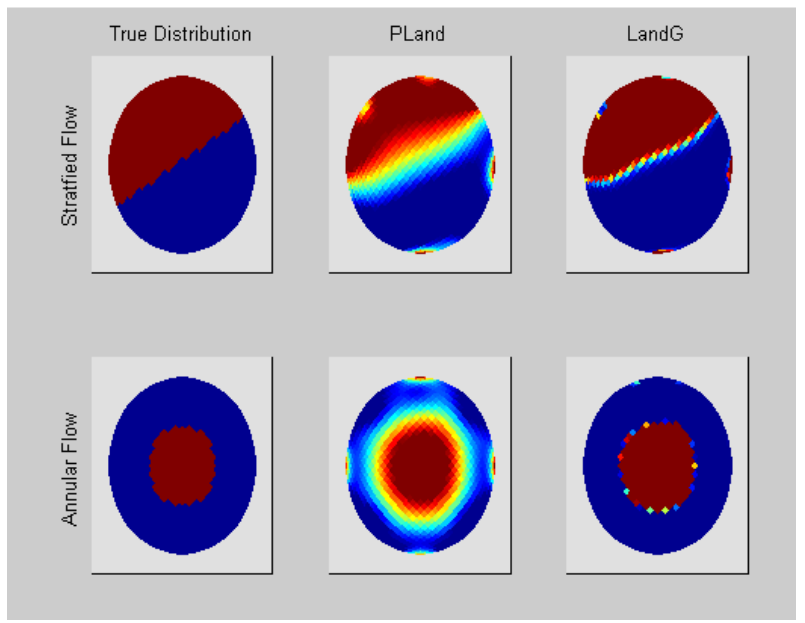

Fig 8: Image Reconstructed Using Projected Landweber Algorithm

Fig. 8 present results from projected Landweber algorithms (PLand) and its modification (PLandG). Again image reconstructed using PLandG are superior compared those reconstructed by PLand.

In general it has been seen that the combination of common algorithms and Gompertz based fitting mechanism proved to produce superior images compared to common algorithms in terms of qualitative evaluation.

Table 1 present quantitative results of the six reconstructed algorithms and their modification using gompertz fitting algorithm.

Table 1. Relative Image Error, Distribution error and Correlation Coefficient for the all Algorithms

\begin{tabular}{|l|l|l|l|l|l|l|l|}
\hline \multirow{2}{*}{ Algorithm } & \multicolumn{2}{|l}{ Relative Image Error } & \multicolumn{2}{l|}{ Distribution Error } & \multicolumn{2}{l|}{ Correlation Coefficient } \\
\cline { 3 - 8 } & Annular & Stratified & Annular & Stratified & Annular & Stratified \\
\hline \multirow{3}{*}{ LBP } & 43.35 & 32.67 & 31.87 & 17.47 & 0.59 & 0.88 \\
\cline { 2 - 8 } & LBPG & 6.97 & 26.77 & 1.12 & 5.99 & 0.99 & 0.92 \\
\hline \multirow{3}{*}{ SVD } & SVD & 57.28 & 41.15 & 34.84 & 50.27 & 0.36 & 0.81 \\
\cline { 2 - 8 } & SVDG & 55.40 & 41.15 & 25.83 & 50.50 & 0.35 & 0.79 \\
\hline \multirow{3}{*}{$\begin{array}{l}\text { Tikhonov } \\
\text { Tikhonov }\end{array}$} & Tikh & 42.89 & 37.05 & 30.44 & 14.98 & 0.74 & 0.85 \\
\cline { 2 - 8 } & TikhG & 21.34 & 38.30 & 3.99 & 9.89 & 0.88 & 0.84 \\
\hline \multirow{3}{*}{\begin{tabular}{l} 
Landweber \\
\cline { 2 - 8 }
\end{tabular}} & ITikhG & 37.63 & 39.33 & 4.92 & 9.60 & 0.86 & 0.84 \\
\cline { 2 - 8 } & LandG & 23.47 & 30.99 & 4.95 & 6.59 & 0.86 & 0.90 \\
\hline \multirow{2}{*}{$\begin{array}{l}\text { Projected } \\
\text { Landweber }\end{array}$} & PLand & 38.05 & 30.94 & 24.18 & 12.14 & 0.75 & 0.90 \\
\cline { 2 - 8 } & PLandG & 22.07 & 28.97 & 4.33 & 6.11 & 0.87 & 0.91 \\
\hline
\end{tabular}


From Table 2, it can be seen that in all cases combination of common algorithms and gompertz fitting algorithms produce better results in terms of relative image error, distribution error and correlation coefficient compared to their counterpart common reconstruction algorithms, except for SVDG algorithm which shows slight variation for the case of DE and CC.

\section{CONCLUSION}

Performance analysis of grey level fitting mechanism based on Gompertz function has been studied. From the results it can be seen that better results (minimum RIE, minimum DE and maximum CC) are obtained when Gompertz data fitting mechanism is applied on the reconstructed images and at insignificant additional computational cost. In this paper LPBG algorithm has been found to be the best overall in terms relative image error, distribution error and correlation coefficient, followed by PLandG, ITikhG, LandG, and SVDG algorithms respectively. In general, it can be said that the application of Gompertz based data fitting mechanism on image reconstructed using ECT system has improved the quality of reconstructed images.

\section{ACKNOWLEDGEMENT}

We wish to extend our appreciation to the Nelson Mandela African Institution of Science and Technology for sponsoring this work and University of Dar es Salaam for granting lab resources.

\section{REFERENCES}

[1] Yang W Q and Peng L 2003 Image reconstruction algorithms for electrical capacitance tomography Meas. Sci. Technol. 14 R1

[2] Marashdeh Q and Fan L 2008 Electrical capacitance tomography-a perspective Ind. Eng. Chem. Res. 47 3708-19

[3] Isaksen $\varnothing 1996$ A review of reconstruction techniques for capacitance tomography Meas. Sci. Technol. 7325

[4] Chandrasekera T and Wang A 2012 A comparison of magnetic resonance imaging and electrical capacitance tomography: An air jet through a bed of particles Powder Technol. 227 86-95

[5] Wang H, Zhu X and Zhang L 2005 Conjugate gradient algorithm for electrical capacitance tomography. Tianjin Daxue Xuebao(J. Tianjin Univ. Sci. Technol.)

[6] Liu S, Fu L and Yang W 2004 Prior-online iteration for image reconstruction with electrical capacitance tomography Sci. Meas. Technol. IEE Proceeding 151 195-200

[7] Rasteiro M and Silva R 2011 Electrical Tomography: a review of Configurations and Applications to Particulate Processes KONA Powder Part. J. 29 67-70

[8] Thorn R 1997 Recent developments in three-phase flow measurement Meas. Sci. Technol. 8691

[9] CHEN Y, GAO B, ZHANG L, CHEN D and YU X 2010 Image reconstruction based on weighted SVD truncation conjugate gradient algorithm for electrical capacitance tomography Opt. Precis. Eng. 3

[10] Yang W Q, Spink D M, York T A and McCann H 1999 An image-reconstruction algorithm based on Landweber's iteration method for electrical-capacitance tomography Meas. Sci. Technol. 101065
[11] Lu G, Peng L, Zhang B and Liao Y 2005 Preconditioned Landweber iteration algorithm for electrical capacitance tomography Flow Meas. Instrum. 16 163-7

[12] Jang J D, Lee S H, Kim K Y and Choi B Y 2006 Modified iterative Landweber method in electrical capacitance tomography Meas. Sci. Technol. 171909

[13] Mou C, Peng L, Yao D and Xiao D 2005 Image reconstruction using a genetic algorithm for electrical capacitance tomography Tsinghua Sci. Technol. 10587 92

[14] Warsito W and Fan L 2003 Neural network multi-criteria optimization image reconstruction technique $(\mathrm{NN}$ MOIRT) for linear and non-linear process tomography Chem. Eng. Process. Process Intensif. 42 663-74

[15] Marashdeh Q and Warsito W 2006 Nonlinear forward problem solution for electrical capacitance tomography using feed-forward neural network Sensors Journal, IEEE 6 pp.441-9

[16] Huang S M, Plaskowski A B, Xie C G and Beck M S 1989 Tomographic imaging of two-component flow using capacitance sensors J. Phys. E. 22 173-7

[17] Xie C G, Huang S M, Beck M S, Hoyle B S, Thorn R, Lenn C and Snowden D 1992 Electrical capacitance tomography for flow imaging: system model for development of image reconstruction algorithms and design of primary sensors IEE Proc. G (Circuits, Devices Syst. 139 89-98

[18] Hua Yan, Chunting Liu and Jing Gao 2004 Electrical capacitance tomography image reconstruction based on singular value decomposition Fifth World Congress on Intelligent Control and Automation (IEEE Cat. No.04EX788) vol 4 (IEEE) pp 3783-6

[19] Tikhonov A and Arsenin V 1977 Solutions of ill-posed problems (Washington, DC : Winston \& Sons)

[20] Wang H, Tang L and Cao Z 2007 An image reconstruction algorithm based on total variation with adaptive mesh refinement for ECT Flow Meas. Instrum. 18 pp.262-7

[21] Björck A 1996 Numerical methods for least squares problems (Siam)

[22] Nombo J 2014 A Grey Level Fitting Mechanism based on Gompertz Function for Two Phase Flow Imaging using Electrical Capacitance Tomography Measurement Int. J. Comput. Appl. 101 7-12

[23] Demidenko E Z 1989 Optimization and regression Moscow. Publ. House "Nauka". Main Ed. Phys. Math Lit. 293

[24] Fletcher R 2013 Practical methods of optimization (John Wiley \& Sons)

[25] Isaksen $\varnothing$ and Nordtvedt J E 1994 An implicit model based reconstruction algorithm for use with a capacitance tomography system Proc. European Concerted Action on Process Tomography, Oporto pp 215-26

[26] Xie C G, Huang S M, Lenn C P, Stott A L and Beck M S 1994 Experimental evaluation of capacitance tomographic flow imaging systems using physical models Circuits, Devices and Systems, IEE Proceedingsvol 141 (IET) pp 357-68 